\title{
Acute antihypertensive effect of self-selected exercise intensity in older women with hypertension: a crossover trial
}

This article was published in the following Dove Press journal: Clinical Interventions in Aging

\author{
Ingrid Bezerra Barbosa \\ Costa (iD) ${ }^{1,2}$ \\ Daniel Schwade ${ }^{2}$ \\ Geovani Araújo Dantas \\ Macêdo (iD) ${ }^{1,2}$ \\ Rodrigo Alberto Vieira \\ Browne iD $^{3,2}$ \\ Luiz Fernando Farias-Junior (DD 3 ,2 \\ Yuri Alberto Freire (D) ${ }^{1,2}$ \\ Júlio Sócrates (iD) 3,2 \\ Kevin F Boreskie iD ${ }^{4,5}$ \\ Todd A Duhamel (iD ${ }^{4,5}$ \\ Eduardo Caldas \\ Costa (iD ${ }^{1-3}$ \\ 'Department of Physical Education, \\ Graduate Program in Physical Education, \\ Federal University of Rio Grande do \\ Norte, Natal, RN, Brazil; ' ${ }^{2}$ epartment of \\ Physical Education, Federal University of \\ Rio Grande do Norte, Natal, RN, Brazil; \\ ${ }^{3}$ Graduate Program in Health Sciences, \\ Federal University of Rio Grande do \\ Norte, Natal, RN, Brazil; ${ }^{4}$ Institute of \\ Cardiovascular Sciences, St Boniface \\ Hospital Albrechtsen Research Centre, \\ Winnipeg, MB, Canada; ${ }^{5}$ Faculty of \\ Kinesiology \& Recreation Management, \\ Health, Leisure, and Human Performance \\ Research Institute, University of \\ Manitoba, Winnipeg, MB, Canada
}

Correspondence: Eduardo Caldas Costa Department of Physical Education, Federal University of Rio Grande do Norte, University Campus, BR I0I, Lagoa Nova, Natal, RN 59.078-970, Brazil Tel/Fax +55843215345I

Email ecc@ufrnet.br
Purpose: Acute reduction in blood pressure (BP) following an exercise session is evidenced in controlled settings with formal supervision in hypertensive older populations. This study investigated the effect of a self-selected exercise (SSE)-intensity session on ambulatory BP in hypertensive older women in a "real-world" setting.

Methods: Twenty inactive older women with hypertension ( $64.9 \pm 4.5$ years) were included in this randomized, controlled, crossover trial. After baseline assessments, participants performed 30 minutes of an SSE-intensity session on an outdoor track and a control session, separated by 7-10 days. Heart rate (HR), rating of perceived exertion (RPE), and affective response were assessed. Ambulatory BP was monitored for 20 hours following both sessions. Paired $t$-tests and generalized estimation were used for data analysis.

Results: Participants exercised at $5.1 \pm 1.1 \mathrm{~km} / \mathrm{h}$, spent $\sim 90 \%$ of the exercise time at moderatevigorous intensity ( $\geq 40 \%$ of heart rate reserve). SSE-intensity session was reported as light (RPE 11.0 \pm 1.5 ) and pleasant (affect 3.4 \pm 1.2 ). SSE-intensity session elicited reductions in systolic BP in the first 6 hours postexercise $(6.0 \mathrm{mmHg}$, CI $2.7-9.3 \mathrm{mmHg} ; P<0.001)$. Average systolic BP in the 20 -hour $(-3.4 \mathrm{mmHg}, \mathrm{CI}-5.9$ to $-0.9 \mathrm{mmHg} ; P=0.010)$ and awake $(-4.0 \mathrm{mmHg}, \mathrm{CI}-6.4$ to $-1.6 \mathrm{mmHg} ; P=0.003)$ periods were lower following SSEintensity session compared to control session. No differences were observed in average systolic BP during asleep period and diastolic BP during the 20-hour awake and asleep periods between the SSE-intensity session and control session $(P>0.05)$.

Conclusion: An SSE-intensity session elicited a reduction in ambulatory systolic BP in inactive older women with hypertension during awake and 20-hour periods. Also, the SSEintensity session was reported as light and pleasant.

Keywords: physical activity, blood pressure, adherence, affect, elderly

\section{Introduction}

Hypertension (HTN) affects more than 1 billion people worldwide, ${ }^{1}$ and its prevalence among older adults is $\sim 70 \%{ }^{2}$ Prevalence of HTN is higher among women than men. ${ }^{2}$ HTN is a major risk factor for coronary artery disease, heart failure, stroke, kidney disease, and mortality. ${ }^{1,2}$ Even in older adults with HTN who are treated with antihypertensive medication(s), high systolic blood pressure (BP) levels are associated with cardiovascular events and all-cause mortality. ${ }^{3}$ Therefore, efforts should be made to decrease BP in older adults with HTN, in order to attenuate the risk of cardiovascular events and mortality. ${ }^{4,5}$

Physical exercise is a cornerstone of nonpharmacological therapies for individuals with HTN, and is widely recommended by guidelines for HTN. ${ }^{6}$ Systematic 
reviews and meta-analyses of randomized controlled trials (RCT) have reported a clear effect of aerobic exercise training for reducing both office ${ }^{7,8}$ and ambulatory ${ }^{9} \mathrm{BP}$ levels, especially in individuals with HTN. A recent network meta-analysis of 391 RCTs confirmed these findings reporting that among individuals with HTN (ie, systolic BP $\geq 140 \mathrm{mmHg}$ ), the systolic BP-lowering effect of aerobic exercise was $\sim 9 \mathrm{mmHg}$, which is similar to the effect of antihypertensive medications. ${ }^{10}$ Taken together, these data strongly endorse aerobic exercise as an antihypertensive therapy.

Exercise prescription in clinical guidelines for the management of HTN have focused on the frequency-intensity-time-and type principle, recommending that hypertensive patients should perform at least 30 minutes of moderate-intensity aerobic exercise (ie, $40 \%-59 \%$ of heart rate $[\mathrm{HR}]$ reserve) most days of the week. ${ }^{6}$ This "exercise dose" elicits both an acute ${ }^{11}$ and a chronic ${ }^{7,9}$ BPlowering effect in individuals with HTN. The acute exercise BP-lowering effect, called postexercise hypotension $(\mathrm{PEH})$, is characterized by reduced $\mathrm{BP}$ values in a postexercise period compared to preexercise and/or by reduced BP values in a postexercise period compared to a control condition. ${ }^{12-14}$ In older adults with HTN, 45 minutes of moderate-intensity aerobic exercise at $50 \%$ of maximal oxygen uptake $\left(\mathrm{VO}_{2 \max }\right)$ can elicit ambulatory PEH that lasts up to 22 hours, including both awake and asleep periods. ${ }^{15}$ The maintenance of lower BP levels through ambulatory $\mathrm{PEH}$ has clinical implications, as these reductions can help prevent cardiovascular events. Therefore, studies characterizing ambulatory $\mathrm{PEH}$, including the magnitude and duration of its presence, following a specific exercise approach may provide valuable information on its efficacy to improve acute BP control in older individuals with HTN.

Although ambulatory $\mathrm{PEH}$ is well documented in older individuals with HTN following formal exercise prescriptions (ie, $\mathrm{HR}$ - or $\mathrm{VO}_{2}$-based) of moderate-intensity aerobic exercise under direct supervision with well-controlled settings, ${ }^{11}$ this is not a realistic scenario for most of these patients. Investigations about the acute effect of feasible exercise approaches on ambulatory BP in "real-world" settings are needed in older individuals with HTN. In this context, self-selected exercise (SSE)-intensity has been suggested as an alternative to prescribed exercise intensity in an effort to improve physical activity levels of inactive populations. ${ }^{16} \mathrm{SSE}$ is characterized by individuals choosing their own exercise intensity according to their preferences. ${ }^{16}$
Older individuals typically self-select an exercise intensity close to their ventilatory threshold, ${ }^{16,17}$ which is within the recommended range proposed for patients with HTN (ie, $\geq 40 \%$ of $\mathrm{HR}$ or $\mathrm{VO}_{2}$ reserve). ${ }^{6}$ In addition, SSEintensity has psychological advantages compared to prescribed exercise intensity, such as higher perceived autonomy, self-efficacy, and affect and lower perceived exertion. ${ }^{18-20}$ When SSE-intensity is performed in an outdoor environment, individuals display higher externally focused attention and affect, lower perceived exertion, and greater enjoyment compared to treadmill exercise. ${ }^{21}$

Therefore, this study aimed to investigate the acute effect of an SSE-intensity session on ambulatory BP in inactive older women with HTN. In addition, HR, affect (ie, feeling of pleasure/displeasure), and perceived exertion during the SSE-intensity session were examined. We hypothesized that an SSE-intensity session would elicit an ambulatory BP-lowering effect, due to participants choosing an intensity within the recommended range proposed for patients with HTN (ie, $\geq 40 \%$ of HR reserve). Furthermore, we hypothesized that participants would report the SSE-intensity as light-moderate and pleasant.

\section{Methods}

\section{Trial design}

This randomized, controlled, crossover clinical trial was designed to assess the effect of a single SSE-intensity session on ambulatory BP in older women with HTN, and is reported in accordance with the CONSORT statement guidelines. ${ }^{22}$ Each participant performed the following procedures during a 4-week period: initial health screening and body composition assessment, assessment of physical activity levels with a pedometer over a 7-day period, maximal graded exercise test, familiarization with ambulatory BP monitoring procedures, familiarization with SSE-intensity session, a single SSE-intensity session and a control session separated by a 7to 10-day period. The study was conducted from August 2016 to May 2017 at the Department of Physical Education, Federal University of Rio Grande do Norte, Brazil. The Institutional Ethics Committee approved the study (protocol 1526048). Participants were informed about all procedures of the study and provided written informed consent.

\section{Participants}

A total of 23 older women with HTN participated in this study. However, only 20 were included in the final 


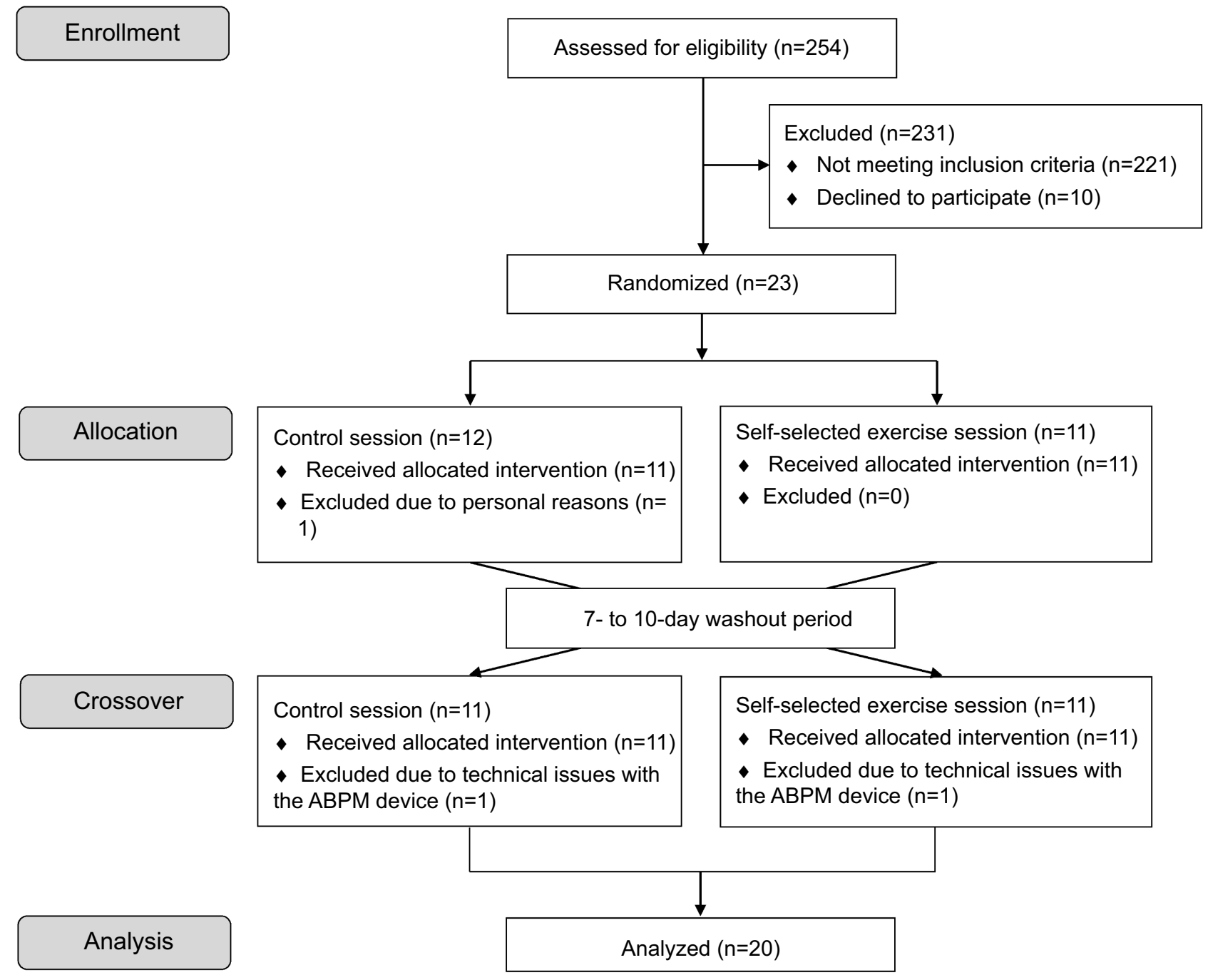

Figure I Flow diagram of the study.

Abbreviation: ABPM, ambulatory blood pressure monitoring.

analysis (see Figure 1). Participants were recruited from the university cardiology outpatient service of the Federal University of Rio Grande do Norte, Brazil. Inclusion criteria were age 60-75 years, previous diagnosis of HTN according to the Brazilian guidelines on $\mathrm{HTN},{ }^{23}$ currently taking antihypertensive medication, no participation in exercise-training programs in last 6 months, "inactive", ie, $<7,500$ steps $/$ day, ${ }^{24}$ no known cardiovascular, metabolic, renal, or respiratory disease, and no musculoskeletal conditions limiting ability to exercise at moderate and vigorous intensity (ie, knee arthritis or pain). Exclusion criteria were: pain or injury limiting ability to exercise during study procedures and changes in antihypertensive medication(s) during the study.

\section{Procedures}

\section{Screening visit}

Participants were screened at their first visit using the Physical Activity Readiness Questionnaire, ${ }^{25}$ medical history, medication use, and physical activity levels (shortversion International Physical Activity Questionnaire). ${ }^{26}$ Clinical examination provided body weight $(\mathrm{kg})$, height (m), resting HR (bpm), and resting BP ( $\mathrm{mmHg}$ ) measurements. Body mass index (BMI) was calculated as weight $(\mathrm{kg})$ divided by height $(\mathrm{m})$ squared. Resting HR was measured after 10 minutes of rest in a supine position in a calm and controlled room $\left(24^{\circ} \mathrm{C}-26^{\circ} \mathrm{C}\right.$ degrees) using an $\mathrm{HR}$ monitor (Polar Electro ${ }^{\circledR}$, Kempele, Finland). Office BP was measured after the resting HR measurement in a seated position using an oscillometric device (Omron ${ }^{\circledR}$ HEM-780-E, Kyoto, Japan) 
in triplicate with 1-minute intervals between each measure. The average value of the last two measures was recorded. ${ }^{23}$ Afterward, the participant's body composition was assessed by dual-energy X-ray absorptiometry (GE Healthcare ${ }^{\circledR}$ Lunar Prodigy Advance, Madison, EUA). At the end of the screening visit, participants were fitted with a wrist pedometer (Omron ${ }^{\circledR}$ HJ-321, Kyoto, Japan) to confirm their physical activity status as assessed by the number of steps accumulated per day over a 1 -week period.

\section{Physical activity level}

Pedometers were individually adjusted for the participants based on their stride length, weight, and height according to the manufacturer's instructions. A total number of steps per day $<7,500$ was used as a criterion to classify the participants as "inactive". ${ }^{24}$ Moreover, the number of steps per day performed at a moderate cadence (ie, 60 steps per minute) ${ }^{27}$ for at least 10 minutes continuously was recorded. This variable was captured to certify that the participants walked less than 30 minutes per day at a moderate cadence (ie, $<1,800$ steps) continuously or in bouts of at least 10 minutes. One week after the first visit, the participants returned to the laboratory to return the wrist pedometer and to perform a maximal graded exercise test.

\section{Maximal graded exercise test}

The participants performed a maximal graded exercise test on a motorized treadmill (Movement ${ }^{\circledR}$ RT350, Pompeia, Brazil) to determine their maximal $\mathrm{HR}\left(\mathrm{HR}_{\max }\right)$, as well as to exclude symptoms and electrocardiographical alterations suggestive of heart disease. Participants were continuously monitored with 12-lead electrocardiography by a cardiologist during the maximal graded exercise test. Following a 2-minute warm-up, an incremental ramp protocol was performed by the participants, starting at a speed of $2.5 \mathrm{~km} /$ $\mathrm{h}$ without grade and increasing $0.3 \mathrm{~km} / \mathrm{h}$ and $1 \%$ grade every minute until volitional exhaustion. The participants' rating of perceived exertion (RPE) was assessed every minute using Borg's (6-20) RPE scale. ${ }^{28}$ BP was measured every minute and immediately following volitional exhaustion. The graded exercise test was considered maximal when the participant reached an RPE $>17$ and HR within $\pm 10 \mathrm{bpm}$ of age-predicted $\mathrm{HR}_{\max }\left(220\right.$-age). ${ }^{29}$ For participants using a $\beta$-blocker, only RPE was used as a criterion of volitional exhaustion. Participants performed the maximal graded exercise test under their regular antihypertensive medication(s). After the exercise test, participants took a shower and had an ambulatory BP monitoring device (Cardios ${ }^{\circledR}$ CardioMapa, São Paulo, Brazil) placed on their nondominant arm to familiarize them with 24-hour ambulatory BP monitoring.

\section{Ambulatory blood pressure monitoring}

Ambulatory BP was assessed three times during the study: during the familiarization session, following the SSEintensity session, and following the control session. The familiarization session was conducted in an effort to avoid an alert reaction, which can happen when wearing an ambulatory BP monitoring device for the first time. This response is more common in women than in men. ${ }^{30}$ This familiarization procedure increased the validity of the ambulatory BP response measurement following the SSEintensity and control sessions. After each experimental session, participants were instructed to take a shower before being fit with the ambulatory BP device (Cardios ${ }^{\circledR}$ CardioMapa, São Paulo, Brazil) on their nondominant arm according to the Brazilian guidelines for ambulatory BP monitoring. ${ }^{31}$ The device was programmed to measure BP every 15 minutes during awake and every 30 minutes during asleep periods. A minimum of 16 and eight BP measurements during awake and asleep periods, respectively, had to be successfully recorded to be included in the final analysis. ${ }^{31}$ Participants came back to the laboratory the following morning after each session so that the BP information could be downloaded to a computer. Data were recorded during a 20-hour period with the awake and asleep periods defined according to each participant. The average values for each period were considered for data analysis. In addition, BP load was calculated during the 20-hour period. This index is defined as the percentage of readings that exceeds the normal BP values in both awake and asleep periods. In the awake period, systolic $\mathrm{BP} \geq 135$ and diastolic $\mathrm{BP} \geq 85 \mathrm{mmHg}$ were considered abnormal. In the asleep period, systolic BP $\geq 120$ and diastolic BP $\geq 70 \mathrm{mmHg}$ were considered abnormal. ${ }^{31}$ All procedures related to ambulatory $\mathrm{BP}$ monitoring were explained to the participants at the screening visit, the familiarization session, and immediately before the experimental sessions, based on the Brazilian guidelines for ambulatory BP monitoring. ${ }^{31}$

\section{Experimental sessions}

The SSE-intensity and control sessions were performed in a randomized order, separated by 7-10 days, on a standard outdoor $400 \mathrm{~m}$ track. All procedures were performed in the 
morning (7-8 am). Participants were instructed to abstain from moderate-vigorous physical activity and caffeinated products on the days of the experimental sessions. Additionally, participants were instructed to abstain from alcohol, maintain their eating habits and sleeping pattern during the 24-hour period prior to the experimental sessions. Participants' resting BP was measured before the experimental sessions following the same procedures as that of the screening visit. They were then fitted with a HR monitor (Polar Electro ${ }^{\circledR}$, Kempele, Finland) to monitor HR responses during both sessions. For the control session, participants remained seated for 40 minutes at the same location where the exercise session was performed. Before the SSE-intensity session, participants were instructed to regulate their own pace using the following statement: "We would like that you exercise for 30 minutes at a pace of your own choosing. You can freely change the pace at any time during the exercise". Following a stretching routine, participants warmed up for 5 minutes to adjust their pace before starting the 30 minutes of SSEintensity session. After, participants cooled down for 5 minutes walking at a slow pace. Whole-body perceived exertion using Borg's (6-20) RPE scale ${ }^{28}$ and basic affect using the Feeling Scale $(-5 /+5)^{32}$ was reported by participants every 10 minutes. They were instructed on use of the RPE scale ${ }^{28}$ and Feeling Scale ${ }^{32}$ at the screening visit, the familiarization visit and immediately before the SSEintensity session. RPE was defined as subjective intensity of effort, strain, and/or fatigue felt during exercise. ${ }^{33}$ Low and high perceptual anchors for Borg's RPE scale were established during the maximal graded exercise test. A rating of 6 (low anchor, "very, very light") was assigned to the lowest exercise intensity, while a rating of 20 (high anchor, "very, very hard") was assigned to the highest exercise intensity. The Feeling Scale ${ }^{32}$ is an 11-point bipolar scale ranging from +5 to -5 recommended by the American College of Sports Medicine to assess affect (ie, feelings of pleasure/displeasure) during exercise. ${ }^{34}$ This scale presents verbal anchors: $-5=$ very bad; $-3=$ bad; $-1=$ fairly bad; $0=$ neutral; +1 fairly good; $+3=$ good; and $+5=$ very good. The participants received standard instructions explaining the Feeling Scale according to Hardy and Rejeski. ${ }^{32}$

Following both experimental sessions, participants were instructed to shower before being fitted with the ambulatory BP device, as previously described. In addition, they were fitted with wrist pedometer to monitor the number of steps they accumulated over the ambulatory BP monitoring period. When they returned in the following day to remove the ambulatory BP monitoring device, they filled out a modified Pittsburgh Sleep Quality Index (PSQI) ${ }^{35}$ to assess the quality and pattern of sleep in the night prior. Two trained investigators (IBBC and DS) conducted the experimental sessions.

\section{Sample size and randomization}

Sample size was calculated using the preliminary data of the primary outcome of the present study (ie, awake systolic ambulatory BP; results obtained from 10 participants); ie, reduction of $3.6 \mathrm{mmHg}(95 \% \mathrm{CI}, 1.0$ to 6.2 $\mathrm{mmHg})$ between the SSE-intensity $(117.7 \pm 8.4 \mathrm{mmHg})$ and control $(121.3 \pm 7.9 \mathrm{mmHg})$ sessions. Based on this preliminary result, 22 participants were needed to power the study, considering $1-\beta$ of $80 \%$ and an alpha of $5 \%$. After all baseline assessments, a computer-based simple randomization (www.graphpad.com) was carried out to determine the order of the experimental sessions. Due to logistics, only the participants were blinded to the order of the experimental sessions.

\section{Statistical analysis}

Data normality was tested using the Shapiro-Wilk test, skewness and kurtosis (score- $z$ : -1.96 to +1.96 ). Results are expressed as mean $\pm \mathrm{SD}$ for the parametric data and median and 25th-75th percentile for the nonparametric data. A paired $t$-test was used to compare the mean values of resting BP, BP load, number of steps, sleep quality score, and ambulatory BP between the sessions. Cohen's $d_{z}$ was used to determine the effect size (ES) of the mean difference. The Hopkins's criteria to interpret the magnitude of the ES were followed: ${ }^{36}<0.2$ is trivial, $0.2-0.5$ is small, $0.6-1.1$ is moderate, $1.2-1.9$ is large, and $2.0-4.0$ is very large. A generalized estimating equation (GEE) was used to assess the condition (2 factors: SSE-intensity session vs control session) by time (3 factors: average values of BP during 1 to 6-hour, 7 to 13-hour, and 14 to 20hour periods) interaction on the ambulatory BP, adjusted for resting $\mathrm{BP}$ values. The GEE model for each outcome was based on the goodness of fit. The normality of the residuals was verified by normal Q-Q plot. For all analyses, a two-tailed $p<0.05$ was considered statistically significant. Data analysis was performed using IBM SPSS Statistics version $25\left(\right.$ IBM $^{\circledR}$, Chicago, USA).

\section{Results}

Table 1 outlines the characteristics of the participants. The mean length of time of since menopause and HTN diagnosis 
Table I Characteristics of participants $(n=20)$

\begin{tabular}{|l|l|}
\hline Variables & Mean \pm SD or median (25th-75th percentile) \\
\hline Age (years) & $64.9 \pm 4.5$ \\
Body mass index $\left(\mathrm{kg} / \mathrm{m}^{2}\right)$ & $29 \pm 4.3$ \\
Fat mass $(\mathrm{kg})$ & $28.9 \pm 7.6$ \\
Fat-free mass $(\mathrm{kg})$ & $39.5 \pm 4.1$ \\
Body fat $(\%)$ & $42.8 \pm 5.3$ \\
Resting SBP $(\mathrm{mmHg})$ & $121.5 \pm 11.2$ \\
Resting DBP $(\mathrm{mmHg})$ & $59.5 \pm 7.9$ \\
20-hour SBP (mmHg) & $119.2 \pm 6.9$ \\
20-hour DBP (mmHg) & $67 \pm 6.1$ \\
Resting HR (bpm) & $62.7 \pm 8$ \\
Maximum HR (bpm) & $154 \pm 14.3$ \\
Steps (number/day) & $5,894(3,718-6,80 \mathrm{I})$ \\
Aerobic steps (number/day)* & $455(0-1,215)$ \\
\hline Antihypertensive medication, $\mathbf{n}(\%)$ & \\
Calcium-channel blocker & $4(20 \%)$ \\
Diuretic & $6(30 \%)$ \\
Angiotensin-receptor antagonist & $14(70 \%)$ \\
$\beta$-blocker & $2(10 \%)$ \\
ACE inhibitor & $2(10 \%)$ \\
\hline
\end{tabular}

Notes: *Steps performed for at least 10 minutes continuously at speed equal or greater than $4 \mathrm{~km} / \mathrm{h}$.

Abbreviations: SBP, systolic blood pressure; DBP, diastolic blood pressure; HR, heart rate.

was $16.9 \pm 7$ and $10.8 \pm 9.1$ years, respectively. The temperature $\left(28{ }^{\circ} \mathrm{C}, 95 \% \mathrm{CI} 27-28{ }^{\circ} \mathrm{C}\right)$ and humidity $(70 \%, 95 \% \mathrm{CI}$ $70-80 \%$ ) of the outdoor track was similar between the SSEintensity and control sessions $(P>0.05)$. Resting systolic $(117.2 \pm 11.8$ vs $119.1 \pm 12.1 \mathrm{mmHg} ; P=0.30)$ and diastolic (70.0 \pm 7.8 vs $71.1 \pm 8.4 \mathrm{mmHg} ; P=0.38) \mathrm{BP}$ values immediately before the SSE-intensity and control sessions were similar. In addition, the participants accumulated the same number of steps $(5,835 \pm 2,627$ vs $5,880 \pm 1,969$ steps/day; $P=0.91)$ in the day of the SSE-intensity and control sessions and experienced similar sleep quality $(3.5,95 \%$ CI $1-6$ vs $4,95 \%$ CI $1-5.8$ arbitrary units; $P=0.76$ ) in the day prior to the sessions.
Table 2 shows the results of the SSE-intensity session. On average, the participants exercised at $5.1 \pm 1.1 \mathrm{~km} / \mathrm{h}$ and spent $\sim 90 \%$ of the exercise time at moderate-to-vigorous intensity. The participants reported the SSE-intensity session as light to moderate (ie, RPE between 10-12) and pleasant (ie, basic affect between 3-4). There were no adverse events during the exercise sessions.

Table 3 shows the results of the ambulatory BP monitoring following the SSE-intensity and control sessions. Average values of systolic BP in the total 20-hour and awake periods were lower following the SSE-intensity session compared to the control session

Table 2 Heart rate, rating of perceived exertion, and affective responses during the self-selected exercise-intensity session in inactive older women with hypertension

\begin{tabular}{|l|l|l|l|l|}
\hline & 0-10 minutes & II-20 minutes & 2I-30 minutes & Mean \\
\hline HR reserve (\%) & $54.3 \pm I I .3$ & $59.4 \pm 9.8$ & $62.9 \pm 11.4$ & $58.8 \pm 9.4$ \\
Light intensity (\%) & $7.8 \pm 22.5$ & $7.9 \pm 23.3$ & $6.8 \pm 23.0$ & $7.5 \pm 22.6$ \\
Moderate intensity (\%)* & $65.9 \pm 40.9$ & $38.7 \pm 39.4$ & $27.0 \pm 38.3$ & $43.8 \pm 34.3$ \\
Vigorous intensity (\%)* & $26.3 \pm 39.3$ & $53.4 \pm 40.7$ & $66.2 \pm 42.3$ & $45.8 \pm 35.6$ \\
RPE (6-20) & $10.4 \pm 1.6$ & $11.2 \pm 1.7$ & $11.5 \pm 1.5$ & $11.0 \pm 1.5$ \\
Affect (-5 to 5) & $3.5 \pm 1.2$ & $3.4 \pm 1.4$ & $3.3 \pm 1.5$ & $3.4 \pm 1.2$ \\
\hline
\end{tabular}

Notes: Values expressed as mean \pm SD. *Percentage of time spent at light intensity ( $<40 \%$ of HR reserve), moderate intensity ( $40 \%-59 \%$ of $H R$ reserve), and vigorous intensity ( $\geq 60 \%$ of HR reserve) during the self-selected exercise-intensity session.

Abbreviations: HR, heart rate; RPE, rating of perceived exertion. 
Table 3 Ambulatory blood pressure monitoring following the self-selected exercise-intensity and control sessions in inactive older women with hypertension

\begin{tabular}{|c|c|c|c|c|c|}
\hline & Control & SSE & $\Delta(95 \% \mathrm{Cl})$ & $P$ & ES \\
\hline \multicolumn{6}{|l|}{ 20-hour } \\
\hline $\mathrm{SBP}(\mathrm{mmHg})$ & $119.2 \pm 6.9$ & $115.8 \pm 7.0$ & $-3.4(-5.9$ to -0.9$)$ & 0.010 & 0.6 \\
\hline $\mathrm{DBP}(\mathrm{mmHg})$ & $67.0 \pm 6.1$ & $66.0 \pm 6.8$ & $-1.0(-2.8$ to 0.7$)$ & 0.219 & 0.3 \\
\hline \multicolumn{6}{|l|}{ Awake } \\
\hline $\mathrm{SBP}(\mathrm{mmHg})$ & $121.6 \pm 7.5$ & $117.6 \pm 8.0$ & $-4.0(-6.4$ to, -1.6$)$ & 0.003 & 0.8 \\
\hline $\mathrm{DBP}(\mathrm{mmHg})$ & $69.7 \pm 6.9$ & $68.1 \pm 8.3$ & $-1.6(-3.5$ to 0.3$)$ & 0.101 & 0.4 \\
\hline \multicolumn{6}{|l|}{ Asleep } \\
\hline $\mathrm{SBP}(\mathrm{mmHg})$ & $114.7 \pm 7.8$ & $111.8 \pm 6.8$ & $-2.9(-6.6$ to 0.8$)$ & 0.114 & 0.4 \\
\hline $\mathrm{DBP}(\mathrm{mmHg})$ & $61.6 \pm 6.1$ & $61.3 \pm 6.4$ & $-0.3(-3.1$ to 2.4$)$ & 0.812 & 0.1 \\
\hline \multicolumn{6}{|l|}{ BP load } \\
\hline SBP (\%) & $17.9(6.7-28.7)$ & $11.7(2.6-19.9)$ & $-6.5(-12.0$ to -1.1$)$ & 0.014 & 0.6 \\
\hline DBP (\%) & $10.3(1.7-19.9)$ & $7.2(1.7-11.9)$ & $-0.6(-5.1$ to 3.9$)$ & 0.327 & 0.1 \\
\hline
\end{tabular}

Notes: Values expressed as mean \pm SD or median (25th-75th percentile). Bold values indicate significant difference $(P<0.05)$.

Abbreviations: SBP, systolic blood pressure; DBP, diastolic blood pressure; SSE, self-selected exercise ES, effect size.

$(P<0.05)$. No differences were observed in the average values of systolic BP during the asleep period and diastolic BP during the 20-hour, awake, and asleep periods between the SSE-intensity and control sessions $(P>0.05)$. The systolic BP load was lower in the SSEintensity session compared to the control session $(P<0.05)$. No differences were found for diastolic BP load between the SSE-intensity and control sessions $(P>0.05)$.

Figure 2 shows the ambulatory BP behavior over the 20 hour period following the SSE-intensity and control sessions. There was significant condition by time interaction for systolic $\mathrm{BP}-\mathrm{W}(2)=7.0 ; P=0.030$. Post hoc analysis revealed a decrease of $6 \mathrm{mmHg}$ (95\% CI $2.7-9.3 \mathrm{mmHg}$ ) in the first 6hour period following the SSE-intensity session compared to the control session $(P=0.004$; Figure 2, panel A). For DBP, there was no significant condition by time interaction $-\mathrm{W}(2)$ $=2.8 ; P=0.252$ (Figure 2, panel B). Table S1 shows the comparison of the absolute values of ambulatory BP over the 20-hour period (ie, 1-6 hours, 7-13 hours, and 14-20 hours).

\section{Discussion}

To the best of our knowledge, this is the first study to investigate the effect of a single SSE-intensity session on ambulatory BP in inactive older women with HTN. The main findings of this study were: (i) the SSE-intensity session elicited a clinically meaningful reduction of 6 $\mathrm{mmHg}$ in the ambulatory systolic BP in the first 6-hour period postexercise. This contributed to the reduction in systolic BP seen during the awake $(4 \mathrm{mmHg}$ ) and the total 20-hour periods $(3.4 \mathrm{mmHg}$ ) when compared to the control session; (ii) the participants met the recommended intensity for aerobic exercise (ie, $\geq 40 \%$ of HR reserve) according to the guidelines for $\mathrm{HTN}^{6}$ and reported the SSE-intensity session as light and pleasant.

Our findings regarding the acute effect of the SSEintensity session on ambulatory BP are in accordance with previous studies that have observed a greater magnitude of ambulatory PEH in the first hours following a HRor $\mathrm{VO}_{2}$-based aerobic exercise session in individuals with HTN. ${ }^{37-39}$ Santos et al ${ }^{38}$ have observed a greater systolic ambulatory PEH in the first 5 hours following 45 minutes of both a light $\left(50 \%\right.$ of $\left.\mathrm{HR}_{\max } ; 7.7 \pm 2.4 \mathrm{mmHg}\right)$ and moderate $\left(75 \%\right.$ of $\left.\mathrm{HR}_{\max } ; 9.4 \pm 2.8 \mathrm{mmHg}\right)$ aerobic exercise session in middle-aged individuals with resistant HTN over 19 hours of ambulatory BP monitoring. Ash et $\mathrm{al}^{39}$ have demonstrated a 10-hour systolic $(5.6 \pm 1.8 \mathrm{mmHg})$ and diastolic $(3.6 \pm 1.3 \mathrm{mmHg})$ BP-lowering effect only in the awake period following 30 minutes of an aerobic exercise session at $60 \%$ of $\mathrm{VO}_{2 \text { peak }}$ in middle-aged individuals with pre- to established stage 1 HTN over 19 hours of ambulatory BP monitoring. Our results suggest that the systolic ambulatory BP-lowering effect following the SSE-intensity session in the first 6 hours improved the acute BP control of the participants; ie, reduced the average values of systolic BP during the awake and 20-hour periods and the systolic BP load.

All participants in the study took antihypertensive medication(s) and presented controlled office $(<140 / 90$ 

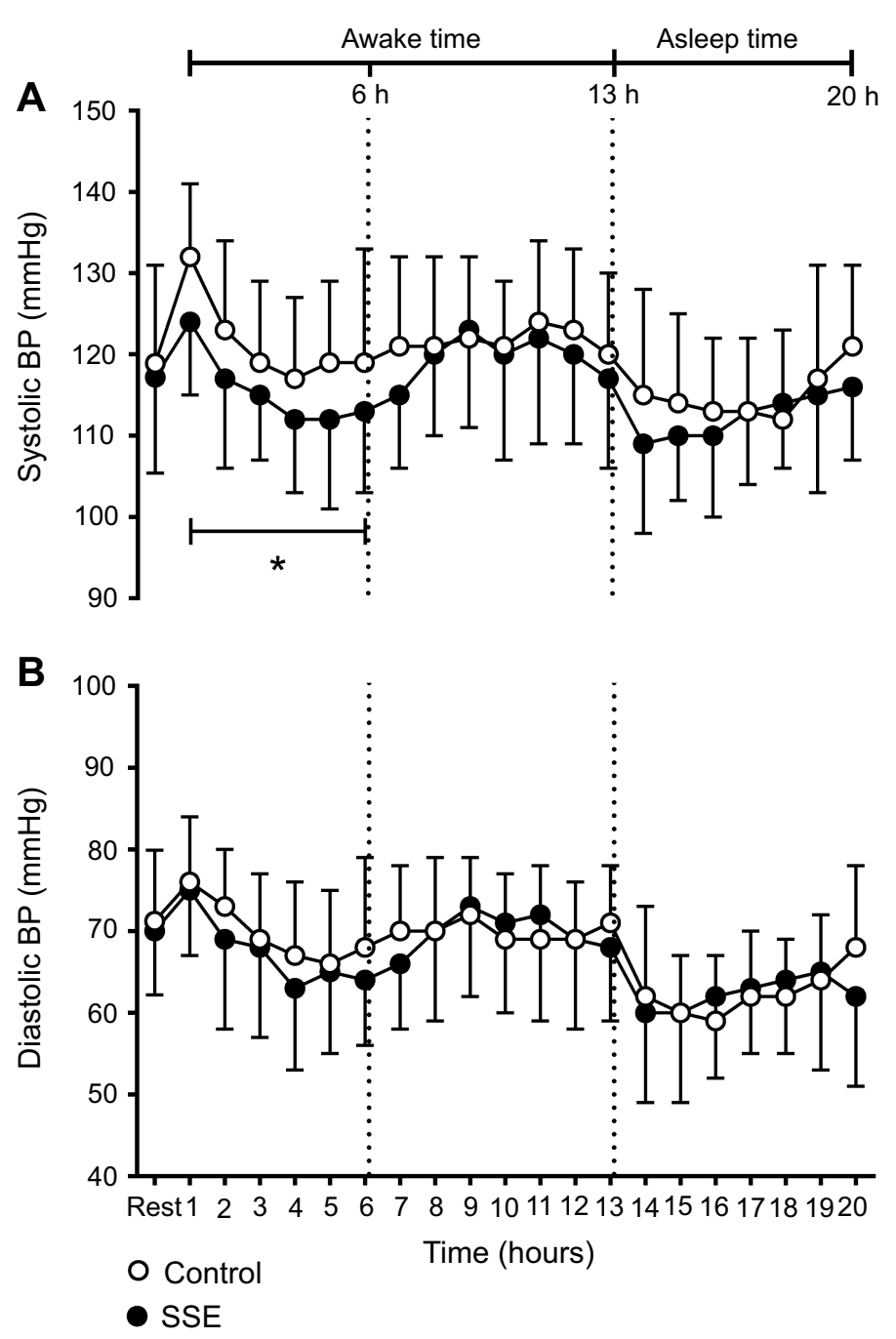

Figure 2 Ambulatory BP monitoring hour-to-hour following the self-selected exercise-intensity (SSE) and control sessions in inactive older women with hypertension. Notes: (A, B) represent systolic and diastolic blood pressure responses, respectively. Values expressed as mean \pm SD. $* P<0.05$ (difference from control session compared to the same period).

Abbreviations: BP, blood pressure; SSE, self-selected exercise.

$\mathrm{mmHg})$ and ambulatory $(<130 / 80 \mathrm{mmHg})$ BP values. Recent data ${ }^{4,40}$ have shown that more intensive systolic BP control (ie, $<120 \mathrm{mmHg}$ ) reduces the risk of fatal and non-fatal cardiovascular events and all-cause mortality compared with traditional target of systolic BP (ie, $<140$ $\mathrm{mmHg}$ ). Despite a well-controlled BP status of the participants, the SSE-intensity session elicited a systolic ambulatory PEH that lasted up to 6 hours. Therefore, the ambulatory PEH observed in our study may have clinical significance in improving the acute BP control of these inactive older women with diagnosed HTN. It should be noted that there is a dose-response relationship between resting $\mathrm{BP}$ levels and the magnitude and duration of PEH. ${ }^{11}$ Thus, it seems reasonable to think that a SSEintensity session could elicit a greater and longer ambulatory PEH in older adults with HTN who present higher BP levels than those included in the present study. Future studies should address this question.

A control condition without a previous exercise session is crucial for the assessment of PEH occurrence as it is supposed to only take sources of BP variation unrelated to exercise and the normal circadian variation of $\mathrm{BP}$ into account. ${ }^{39,41}$ In addition, the ambulatory BP monitoring during a control condition demonstrates the cardiovascular load during daily activities, including sedentary behaviors in different positions (ie, seated and supine), posture changes with orthostatic stress, walking and other physical activities. Thus, the ambulatory systolic BP-lowering effect of $\sim 4 \mathrm{mmHg}$ observed in the awake and 20-hour periods and the reduction of $\sim 6 \%$ in the systolic BP load 
following the SSE-intensity session, as compared to the control condition, may play a role in attenuating the overload on the cardiovascular system imposed by sedentary behaviors and light-intensity activity (ie, <4 METs). It should be noted that older adults spend more than $95 \%$ of their awake time in sedentary behaviors and light physical activities. ${ }^{42}$

Our data support the idea that a SSE-intensity session elicits a systolic ambulatory $\mathrm{PEH}$, which was similar to previous studies that have used traditional $\mathrm{HR}$ - or $\mathrm{VO}_{2^{-}}$ based exercise prescription approaches. ${ }^{11}$ Overall, the studies have reported that middle-aged and older individuals self-select an exercise intensity close to ventilatory threshold $^{16-18}$ and within the recommended range proposed for apparently healthy individuals and patients with HTN (ie moderate to vigorous; $\geq 40 \%$ of $\mathrm{HR}$ or $\mathrm{VO}_{2}$ reserve). ${ }^{6}$ In our study, the participants exercised at a brisk walking pace $(5.1 \pm 1.1 \mathrm{~km} / \mathrm{h} ; \sim 105$ steps/minute), spent $\sim 90 \%$ of the exercise session at moderate to vigorous intensity, and presented a mean HR response equivalent to $58.8 \%$ of HR reserve. Therefore, it seems clear that the acute ambulatory BP-lowering effect observed in our study is associated with the exercise intensity level achieved by the participants during the SSE-intensity session. This finding is consistent with a previous study that observed an ambulatory $\mathrm{PEH}$ following a $\mathrm{VO}_{2}$-based moderate intensity aerobic exercise session in older individuals with HTN. ${ }^{15}$

Despite the participants having presented HR responses consistent with moderate to vigorous exercise intensity, they reported a RPE consistent with light physical activity (ie, RPE from 10 to 11) during the SSE-intensity session. Exercise at a self-selected pace elicits a high perceived autonomy and pleasant feelings, which may have contributed to attenuate a perceived exertion consistent with a moderate-intensity aerobic exercise. ${ }^{20}$ Moreover, the various stimuli present in an outdoor setting seems to reduce internal attention focus (eg, increased HR, breath, pain) and increase more externally focused attention. ${ }^{21}$ Thus, individuals can report a lower RPE than expected for a moderate to vigorous exercise intensity. This aspect seems to be important for older adults, especially those with a low self-efficacy for exercise, as this is an important factor associated with lack of engagement in regular physical activity by this population. ${ }^{43}$

The participants reported the SSE-intensity session as pleasant. This can be partially explained by the magnitude of the metabolic stress imposed on the participants during the exercise. Overall, the individuals self-select an exercise intensity close to ventilatory threshold. ${ }^{16-18}$ Lind et al ${ }^{18}$ reported that SSE-intensity is characterized by a stable and positive affective state, which is often maintained close to, but below, the ventilatory threshold. Therefore, SSE-intensity seems to be an interesting approach for increasing physical activity levels in inactive older women with HTN. In this context, it is important to highlight that even among hypertensive patients taking antihypertensive medication(s) and with well-controlled BP levels, those who are physically active have a lower cardiovascular risk than their inactive peers. ${ }^{4}$

From a practical perspective, SSE-intensity seems to be a simple exercise approach for improving acute BP control of inactive older adults with HTN through the maintenance of systolic ambulatory PEH. In addition, it seems more feasible than the traditional $\mathrm{HR}$ - or $\mathrm{VO}_{2}$-based aerobic exercise prescription in community-based settings where there may be no formal supervision. Therefore, it seems reasonable that clinicians and healthcare providers consider providing instructions for SSE-intensity for older adults with HTN, especially for those who are inactive and unfit.

Despite our novel findings, some limitations of this study must be mentioned. First, our sample was composed of medicated hypertensive older women with wellcontrolled office and ambulatory BP status. Our findings may not be generalizable to other hypertensive populations. Second, only HR responses were monitored as a physiological marker of the exercise intensity, therefore, it was not possible to characterize the SSE-intensity session according to $\mathrm{VO}_{2}$ and metabolic domains (below, at or above the ventilatory threshold). Third, despite the instruction for the participants to abstain from alcohol and to maintain their eating habits in the 24-hour period before the experimental sessions, the compliance with this instruction was not assessed. However, it should be noted that important confounding factors were monitored in order to improve the internal validity of this study, such as the familiarization with the ambulatory BP monitoring device, the measurement of the quality and patterns of sleep in the night prior to the experimental sessions, and the assessment of the physical activity levels by pedometer over the 20 hours of ambulatory BP monitoring following the experimental sessions.

\section{Conclusion}

A single SSE-intensity session lasting 30 minutes elicited a reduction in ambulatory systolic BP in inactive older 
women with HTN in the first 6-hour period post exercise, which contributed to the reduction of systolic BP during the awake and 20-hour periods. This SSEintensity approach met the recommended intensity for aerobic exercise (ie, moderate to vigorous) according to the guidelines for HTN. In addition, it was perceived as light and pleasant. Therefore, SSE-intensity should be considered as a simple alternative to encourage inactive older women with HTN become more physically active. Future studies should investigate whether SSE-intensity sessions can elicit a chronic ambulatory BP-lowering effect and improve health-related physical fitness in older adults with HTN.

\section{Acknowledgments}

Master's degree scholarship granted by Coordination for the Improvement of Higher Education Personnel (CAPES, Brazil) to IBBC. All procedures were approved by the Ethics Committee of the Federal University of Rio Grande do Norte (protocol 1526048) and were carried out in line with the Declaration of Helsinki. The authors thank the cardiologist Bruno Erick de Barros Lucena to conduct the graded exercise test with the participants of the study.

\section{Disclosure}

The authors report no conflicts of interest in this work.

\section{References}

1. Fisher NDL, Curfman G. Hypertension-A public health challenge of global proportions. Jama. 2018;320(17):1757. doi:10.1001/ jama.2018.16760

2. Benjamin EJ, Virani SS, Callaway CW, et al. Heart disease and stroke statistics-2018 update: a report from the American Heart Association. Circulation. 2018;137:12. doi:10.1161/CIRCULATIONAHA.117. 031422

3. Zhou D, Xi B, Zhao M, Wang L, Veeranki SP. Uncontrolled hypertension increases risk of all-cause and cardiovascular disease mortality in US adults: the NHANES III Linked Mortality Study. Sci Rep. 2018;8 (1):9418. doi:10.1038/s41598-018-27377-2

4. Williamson JD, Supiano MA, Applegate WB, et al. Intensive vs standard blood pressure control and cardiovascular disease outcomes in adults aged $\geq 75$ years. Jama. 2016;315(24):2673. doi:10.1001/jama.2016.7050

5. Jaeger BC, Anstey DE, Bress AP, et al. Cardiovascular disease and mortality in adults aged $\geq 60$ years according to recommendations by the American College of Cardiology/American Heart Association and American College of Physicians/American Academy of Family Physicians. Hypertension. 2019;73(2):327-334. doi:10.1161/ HYPERTENSIONAHA.118.12291

6. Pescatello LS, MacDonald HV, Lamberti L, Johnson BT. Exercise for hypertension: a prescription update integrating existing recommendations with emerging research. Curr Hypertens Rep. 2015;17(11):87. doi:10.1007/s11906-015-0600-y
7. Cornelissen VA, Smart NA. Exercise training for blood pressure: a systematic review and meta-analysis. $J$ Am Heart Assoc. 2013;2(1): e004473. doi:10.1161/JAHA.112.004473

8. Costa EC, Dantas TCB, Farias-Júnior LF. et al. Reproducibility of ambulatory blood pressure after high-intensity interval training sessions in healthy individuals. Blood Press Monit;2018. 1. doi:10.1097/ MBP.0000000000000345

9. Sosner P, Guiraud T, Gremeaux V, Arvisais D, Herpin D, Bosquet L. The ambulatory hypotensive effect of aerobic training: a reappraisal through a meta-analysis of selected moderators. Scand J Med Sci Sports. 2017;27(3):327-341. doi:10.1111/sms.12661

10. Naci H, Salcher-Konrad M, Dias S, et al. How does exercise treatment compare with antihypertensive medications? A network meta-analysis of 391 randomised controlled trials assessing exercise and medication effects on systolic blood pressure. Br J Sports Med. Epub 2018 Dec 18. bjsports-2018-099921.

11. Carpio-Rivera E, Moncada-Jiménez J, Salazar-Rojas W, Solera-Herrera A. Acute effects of exercise on blood pressure: a meta-analytic investigation. Arq Bras Cardiol. 2016. doi:10.5935/abc.20160064

12. Costa E, Dantas T, de Farias Junior L, et al. Inter- and intra-individual analysis of post-exercise hypotension following a single bout of high-intensity interval exercise and continuous exercise: a pilot study. Int J Sports Med. 2016;37(13):1038-1043. doi:10.1055/s-0042-112029

13. Dantas TCB, Farias Júnior LF, Frazão DT, et al. A single session of low-volume high-intensity interval exercise reduces ambulatory blood pressure in normotensive males Ambulatory blood pressure and high-intensity interval exercise. $J$ Strength Cond Res. 2017;1 (8):2263-2269. doi:10.1519/JSC.0000000000001688

14. Costa EC, Hay JL, Kehler DS, et al. Effects of high-intensity interval training versus moderate-intensity continuous training on blood pressure in adults with pre- to established hypertension: a systematic review and meta-analysis of randomized trials. Sports Med. 2018;48 (9):2127-2142. doi:10.1007/s40279-018-0944-y

15. Brandão Rondon MUP, Alves MJNN, Braga AMFW, et al. Postexercise blood pressure reduction in elderly hypertensive patients. J Am Coll Cardiol. 2002;39(4):676-682.

16. Ekkekakis P. Let them roam free? Sport Med. 2009;39(10):857-888. doi:10.2165/11315210-000000000-00000

17. Smith AE, Eston R, Tempest GD, Norton B, Parfitt G. Patterning of physiological and affective responses in older active adults during a maximal graded exercise test and self-selected exercise. Eur J Appl Physiol. 2015;115(9):1855-1866. doi:10.1007/s00421-015-3167-z

18. Lind E, Joens-Matre RR, Ekkekakis P. What intensity of physical activity do previously sedentary middle-aged women select? Evidence of a coherent pattern from physiological, perceptual, and affective markers. Prev Med (Baltim). 2005;40(4):407-419. doi:10.1016/j.ypmed.2004.07.006

19. Ekkekakis P, Lind E. Exercise does not feel the same when you are overweight: the impact of self-selected and imposed intensity on affect and exertion. Int $J$ Obes (Lond). 2006;30(4):652-660. doi:10.1038/sj.ijo.0803052

20. Ekkekakis P, Parfitt G, Petruzzello SJ. The pleasure and displeasure people feel when they exercise at different intensities decennial update and progress towards a tripartite rationale for exercise intensity prescription. Sport Med. 2011;41(8):641-671. doi:10.2165/ 11590680-000000000-00000

21. Krinski K, Machado DGS, Lirani LS, et al. Let's walk outdoors! selfpaced walking outdoors improves future intention to exercise in women with obesity. J Sport Exerc Psychol. 2017;39(2):145-157. doi:10.1123/jsep.2016-0220

22. Boutron I, Altman DG, Moher D, Schulz KF, Ravaud P. CONSORT statement for randomized trials of nonpharmacologic treatments: a 2017 update and a CONSORT extension for nonpharmacologic trial abstracts. Ann Intern Med. 2017;167(1):40. doi:10.7326/M17-0046 
23. Malachias MVB. 7th Brazilian guideline of arterial hypertension: presentation. Arq Bras Cardiol. 2016;107(3):1-83. doi:10.5935/ abc. 20160151

24. Tudor-Locke C, Hatano Y, Pangrazi RP, Kang M. Revisiting "how many steps are enough? Med Sci Sports Exerc. 2008;40(7 Suppl): S537-S543. doi:10.1249/MSS.0b013e31817c7133

25. Warburton DER, Gledhill N, Jamnik VK, et al. Evidence-based risk assessment and recommendations for physical activity clearance: consensus document 20111 This paper is one of a selection of papers published in this special issue, entitled evidence-based risk assessment and recommendations for physi. Appl Physiol Nutr Metab. 2011;36(S1):S266-S298. doi:10.1139/h11-062

26. Craig CL, Marshall AL, Sjojtrom M, et al. International physical activity questionnaire: 12-country reliability and validity. Med $\mathrm{Sci}$ Sport Exerc. 2003;35(8):1381-1395. doi:10.1249/01.MSS.000007 8924.61453.FB

27. Tudor-Locke C, Han H, Aguiar EJ, et al. How fast is fast enough? Walking cadence (steps/min) as a practical estimate of intensity in adults: a narrative review. Br J Sports Med. 2018;52(12):776-788. doi:10.1136/bjsports-2017-097628

28. Borg G. Perceived exertion as an indicator of somatic stress. Scand J Rehabil Med. 1970;2(2):92-98.

29. Ferguson B. ACSM's guidelines for exercsie testing and prescription 9th Ed. J Can Chiropr Assoc. 2014;58(3):328.

30. Ash G, Walker T, Olson K, et al. Reproducibility of ambulatory blood pressure changes from the initial values on two different days. Clinics. 2013;68(12):1509-1515. doi:10.6061/clinics/2013(12)06

31. Sociedade Brasileira de Cardiologia (SBC), Sociedade Brasileira de Hipertensão (SBH), Sociedade Brasileira de Nefrologia (SBN). [V Guidelines for ambulatory blood pressure monitoring (ABPM) and III Guidelines for home blood pressure monitoring (HBPM)]. Arq Bras Cardiol. 2011;97(3 Suppl 3):1-24.

32. Hardy CJ, Rejeski WJ. Not what, but how one feels: the measurement of affect during exercise. J Sport Exerc Psychol. 1989;11:204-317. doi:10.1123/jsep.11.3.304

33. Pageaux B. Perception of effort in exercise science: definition, measurement and perspectives. Eur J Sport Sci. 2016;16(8):885-894. doi:10.1080/17461391.2016.1188992

34. Garber CE, Blissmer B, Deschenes MR, et al. Quantity and quality of exercise for developing and maintaining cardiorespiratory, musculoskeletal, and neuromotor fitness in apparently healthy adults. Med Sci Sport Exerc. 2011;43(7):1334-1359. doi:10.1249/MSS.0b013e318213fefb
35. Buysse DJ, Reynolds CF, Monk TH, Berman SR, Kupfer DJ. The pittsburgh sleep quality index: a new instrument for psychiatric practice and research. Psychiatry Res. 1989;28(2):193-213.

36. Hopkins WG. A scale of magnitudes for effect statistics. 2002. Available from: https://www.sportsci.org/resource/stats/effectmag.html. Accessed June 7, 2019.

37. Ciolac EG, Guimarães GV, D'Àvila VM, Bortolotto LA, Doria EL, Bocchi EA. Acute effects of continuous and interval aerobic exercise on 24-h ambulatory blood pressure in long-term treated hypertensive patients. Int J Cardiol. 2009;133(3):381-387. doi:10.1016/j.ijcard.2008.02.005

38. Santos LP, Moraes RS, Vieira PJC, et al. Effects of aerobic exercise intensity on ambulatory blood pressure and vascular responses in resistant hypertension. $J$ Hypertens. 2016;34(7):1317-1324. doi:10.1097/HJH.0000000000000961

39. Ash GI, Taylor BA, Thompson PD, et al. The antihypertensive effects of aerobic versus isometric handgrip resistance exercise. J Hypertens. 2017;35(2):291-299. doi:10.1097/HJH.0000000000001176

40. Ettehad D, Emdin CA, Kiran A, et al. Blood pressure lowering for prevention of cardiovascular disease and death: a systematic review and meta-analysis. Lancet. 2016;387(10022):957-967. doi:10.1016/ S0140-6736(15)01225-8

41. de Brito LC, Rezende RA, Da Silva Junior ND, et al. Post-exercise hypotension and its mechanisms differ after morning and evening exercise: a randomized crossover study. Shea SA, ed. PLoS One. 2015; 10(7):e0132458. doi:10.1371/journal.pone.0132458

42. Harvey JA, Chastin SFM, Skelton DA. How sedentary are older people? A systematic review of the amount of sedentary behavior. J Aging Phys Act. 2015;23(3):471-487. doi:10.1123/japa.2014-0164

43. Jefferis BJ, Sartini C, Lee I-M, et al. Adherence to physical activity guidelines in older adults, using objectively measured physical activity in a population-based study. BMC Public Health. 2014;14(1):382. doi:10.1186/1471-2458-14-382

44. Brown RE, Riddell MC, Macpherson AK, Canning KL, Kuk JL. The joint association of physical activity, blood-pressure control, and pharmacologic treatment of hypertension for all-cause mortality risk. Am J Hypertens. 2013;26(8):1005-1010. doi:10.1093/ajh/hpt063 


\section{Supplementary material}

Table SI Ambulatory blood pressure monitoring per period following self-selected exercise-intensity and control sessions in inactive older women with hypertension

\begin{tabular}{|c|c|c|c|}
\hline & \multicolumn{2}{|l|}{ Awake } & \multirow{2}{*}{$\begin{array}{l}\text { Asleep } \\
14-20 \text { hours }\end{array}$} \\
\hline & I-6 hours & $7-13$ hours & \\
\hline \multicolumn{4}{|c|}{ SBP (mmHg) } \\
\hline Control & $121.4 \pm 7.5$ & $121.6 \pm 7.9$ & II $5.4 \pm 7.5$ \\
\hline SSE & II $5.4 \pm 7.5^{*}$ & $119.5 \pm 9.4$ & $112.5 \pm 6.8$ \\
\hline$\Delta(95 \% \mathrm{Cl})$ & $6.0(2.7-9.3)$ & 2.1 ( -0.5 to 4.7$)$ & $2.5(-1.1$ to 6.1$)$ \\
\hline \multicolumn{4}{|c|}{ DBP (mmHg) } \\
\hline Control & $69.6 \pm 7.3$ & $69.6 \pm 6.7$ & $62.2 \pm 5.8$ \\
\hline SSE & $67.1 \pm 8.2$ & $68.9 \pm 8.7$ & $62.0 \pm 6.3$ \\
\hline$\Delta(95 \% \mathrm{Cl})$ & $2.5(0.2-4.7)$ & $0.7(-1.4$ to 2.8$)$ & $0.1(-2.4$ to 2.6$)$ \\
\hline
\end{tabular}

Notes: Values expressed as means \pm SD and $95 \%$ Cls. $* P<0.05$ compared to the same period of the control session.

Abbreviations: SBP, systolic blood pressure; DBP, diastolic blood pressure; SSE, self-selected exercise.

\section{Publish your work in this journal}

Clinical Interventions in Aging is an international, peer-reviewed journal focusing on evidence-based reports on the value or lack thereof of treatments intended to prevent or delay the onset of maladaptive correlates of aging in human beings. This journal is indexed on PubMed Central, MedLine, CAS, Scopus and the Elsevier
Bibliographic databases. The manuscript management system is completely online and includes a very quick and fair peer-review system, which is all easy to use. Visit http://www.dovepress.com/ testimonials.php to read real quotes from published authors. 